\title{
Separation characteristics between time domain and frequency domain of wireless power communication signal in wind farm
}

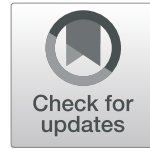

Jie Wan ${ }^{1,2,3}$, Kun Yao $^{4 *}$, E. Peng ${ }^{1 *}$, Yong $\mathrm{CaO}^{4}$, Yuguang $\mathrm{NIU}^{5}$ and Jilai $\mathrm{Yu}^{2,6}$

\begin{abstract}
Understanding the intrinsic characteristics of wind power is important for the safe and efficient parallel function of wind turbines in large-scale wind farms. Current research on the spectrum characteristics of wind power focuses on estimation of power spectral density, particularly the structural characteristics of Kolmogorov's scaling law. In this study, the wavelet Mallat algorithm, which is different from the conventional Fourier transform, with compactly supported characteristics is used to extract the envelope of the signal and analyze the instantaneous spectral characteristics of wind power signals. Then, the theory for the change in the center frequency of the wind power is obtained. The results showed that within a certain range, the center frequency decreases as the wind power increases by using enough wind farm data. In addition, the center frequency remains unchanged when the wind power is sufficiently large. Together with the time domain characteristics of wind power fluctuation, we put forward the time-frequency separation characteristics of wind power and the corresponding physical parameter expressions, which corresponds to wind speed's amplitude and frequency modulation characteristics. Lastly, the physical connotation of the time-frequency separation characteristics of wind power from the perspective of atmospheric turbulent energy transport mechanism and wind turbine energy transfer mechanism is established.
\end{abstract}

Keywords: Power of wind turbine, FFT, Mallat, Instantaneous power spectrum, Time domain fluctuation, Timefrequency separation, Turbulent energy transport, Turbine energy transform

\section{Introduction}

Currently, utilizing wind energy to its complete potential has been the goal of energy development in all countries worldwide, especially in China. Although this initiative had a slow start, it has rapidly developed [1-3]. However, the variable output brings new challenges to the safe and efficient operation of the power system [4-6], especially in China. The structural differences of energy have resulted in an increasing number of high-power coal-fired units to perform deep and variable load operation and consequently increasing the probability of fault occurrences $[7,8]$. Therefore, understanding wind power characteristics is extremely important to understand not only the requirements of wind farm sits but also for the

\footnotetext{
* Correspondence: 19B353009@stu.hit.edu.cn; epeng@hit.edu.cn

${ }^{4}$ School of Mechanical Engineering and Automation, Harbin Institute of

Technology, Shenzhen 518055, China

${ }^{1}$ Fundamental Space Science Research Center, Harbin Institute of

Technology, Harbin 150001, Heilong, China

Full list of author information is available at the end of the article
}

real-time scheduling and optimization control of new energy power systems $[9,10]$.

In general, the uncertain characteristics of wind energy include randomness, volatility, and intermittency. The study on the time domain characteristics is an important aspect in exploring the variable wind power fluctuations. In [11-13], a model was developed for the fluctuations in wind power, which was based on measured data, and the model accuracy was verified. Because wind turbines channel wind to generate winder power, studying the characteristics of wind speed is fundamental. In [14], a large number of actual wind speed data was used for the range of wind speed fluctuations in wind farms to correct the quantitative characterization model to develop and improve it as per the IEC standard. On this basis, literature [15] further evaluated the frequency modulation capability and explored the application of the quantitative characterization model for a range of wind speed fluctuations. In [16], a modeling strategy that 
can quantitatively describe the rate of wind speed fluctuation based on a variable differential model was proposed. Therefore, the understanding of intermittent quantitative description models is also the basic concern of this study [17, 18]. In [19], the quantitative characterization model of the intermittency of wind speed based on abrupt changes in the duty cycle from the intermittent nature of atmospheric turbulence was developed. Moreover, this method can be applied to the intermittent quantitative characterization of wind power [20]. In addition, although the wind power prediction model is constantly improving, because of the uncertainty of wind power, and incorporating some intrinsic characteristics of wind power, the model accuracy can still improve and the actual prediction error is inevitable [21]. Therefore, many scholars are also analyzing the error [22-24].

The power spectral density of a random signal is used to describe the relationship between the energy characteristics of a signal and the frequency. Therefore, the power spectrum analysis of wind power is also an important aspect of wind power characteristics research. Further study has been carried out very early, especially in the analysis of the spectral characteristics of wind. In 1941, a former Soviet Union mathematician named Andrei N. Kolmogorov proposed the Kolmogorov hypothesis [25]. When the Reynolds number is sufficiently large, there is a region with a high wave number, in which the turbulence satisfies local uniformity and isotropy. In addition, the turbulence characteristics are only determined by the energy dissipation rate and the molecular viscosity coefficient. There is also an inertial subregion in the local isotropic region, where the turbulence characteristics are only determined by the energy dissipation rate. Based on this assumption, Kolmogorov came up with the famous "- $5 / 3$ law." After this, many domestic and overseas scholars have verified and improved the Kolmogorov's "- $5 / 3$ law" [26, 27]. The results show that the energy spectrum of the near-surface turbulence satisfies the Kolmogorov spectral distribution theory under certain conditions. However, there is a deviation in the exponent values around $-5 / 3$ because of different geographical and climatic factors. Nevertheless, the energy spectrum exponentially decreases in a certain frequency domain. The energy spectrum characteristics of the wind turbine output power are studied further on this basis $[28,29]$. In [30], wind farm power fluctuations and spatial sampling of turbulent boundary layers are presented. The experimental results show that the frequency spectrum of the total wind farm power follows a power law with a slope between $-5 / 3$ and -2 , and up to frequencies lower than seen for any individual turbine model. However, the results of the above research state that the conventional power spectrum characteristics, i.e., the variation law of wind power signal's frequency, were compared and analyzed with those of the structural characteristics of the Kolmogorov scaling law. Moreover, the above studies consider the time domain and the frequency domain in isolation.

In addition, the IEC standard improved the linear turbulence model for wind farm turbines' integrated fatigue loading by combining ambient turbulence and wakes [31]. In fact, this is the amplitude modulation (AM) effect. In [32], experiments in the wind tunnel revealed the AM effect of all three velocity components. The center frequency is another vital parameter to describe wind speed uncertainty as well, which is usually estimated for classical wind speed power spectrum [33]. In the signal processing field, there are three kinds of modulation, including AM, FM (frequency modulation), and PM (phase modulation) [34]. And the current research is also focused on the scale rate of the wind speed frequency spectrum without combining the time domain with frequency domain.

In this study, the difference in the characteristics between the time domain and frequency domain of wind power signal is studied. This paper is organized as follows. In Section 2, we introduced methods for obtaining and analyzing transient spectral features and analyzed the principles and advantages of the Mallat algorithm with compact support. In Section 3, we used the wavelet Mallat algorithm to extract the envelope of the signal and analyze the instantaneous spectral characteristics of the actual wind power data. Combined with the time-domain uncertainty of wind power fluctuation time-frequency separation characteristics we gave the corresponding physical parameter expressions, including wind speed corresponding characteristics. In Section 4, it shows that the physical connotation of time-frequency separation characteristics of wind power from the perspective of atmospheric turbulence energy transport mechanism and wind turbine energy transfer mechanism.

\section{Method}

\subsection{Power spectrum estimation based on Fourier transform [28, 30, 35]}

The wind signal is an indeterminate random signal. According to the stochastic process theory, statistics such as mean, mean square, correlation function, and power spectral density function can be used to describe the characteristics of a random process or a random signal. Moreover, the spectrum characteristic analysis of the actual signal can be performed by using the Fourier transform method.

Since an arbitrary function $x(t)$ can be decomposed into the sum of an infinite number of sinusoidal signals of different frequencies, this is similar to the phenomenon in which white light is refracted by prisms and dispersed into light of different colors. Comparing the Fourier transform 
with the refractive index of light, it is apparent that the Fourier transform method is equivalent to the triangular prism in the spectral analysis. The signal $x(t)$ is equivalent to a beam of white light. In addition, after $x(t)$ is analyzed by using Fourier transform, the "spectrum" of the resulting signal is equivalent to the spectrum, as shown in Fig. 1.

Power spectrum estimation (PSD) is an important research subject matter in digital signal processing. PSD can be divided into classical PSD (non-parametric estimation) and modern PSD (parameter estimation). Classical PSD includes correlation function, period diagram, Bartlett, and Welch period diagram methods. Modern PSD includes the maximum entropy spectrum analysis (AR model), Pisarenko harmonic decomposition, Prony extraction pole method, Prony spectral line decomposition, and Capon maximum likelihood.

When applying PSD to discrete sampling, the general solutions for the two types of errors and the trend term problem are as follows:

(1) After sampling, the spectral function $S_{n}$ is changed to $S_{T}(n)$ :

$$
S_{T}(n)=\int_{-\infty}^{\infty} S(f) \frac{\sin \pi T(n-f)}{\pi(n-f)} d f
$$

The modified spectrum $S_{T}(n)$ has a false highfrequency component. In general, the higher $T$ is, the closer $S_{T}(n)$ is to the true spectrum $S_{n}$. The smaller $T$ is, the larger the difference is between $S_{T}(n)$ and $S_{n}$, and the greater the impact of leakage is. Therefore, the spectrum can be smoothed by a suitable energy window $W(n)$, which is a weighted average method to reduce leakage.

(2) Two aspects of distortion occur when sampling at equal intervals $\Delta t$ as sampling intervals: On the one hand, the spectrum will reduce from $(-\infty, \infty)$ to $\left(-\frac{n_{f}}{2}, \frac{n_{f}}{2}\right),|n| \geq \frac{n_{f}}{2}$, and $n_{f}=\frac{1}{\Delta t}$. On the other hand, the spectrum becomes a folding spectrum in the reduced range, and the aliasing frequency is $\frac{n_{f}}{2}=\frac{1}{2 \Delta t}$. Therefore, we generally choose the appropriate $\Delta t\left(\Delta t<\frac{1}{2 n_{c}}\right.$, where $n_{c}$ is the highest frequency and $\Delta t$ is the sampling interval) to avoid aliasing.

(3) The trend term is a problem that must be carefully considered in the turbulence analysis. It usually needs to eliminate the large-scale influence through low-pass mathematical filtering because the correlation function and spectrum obtained by detrending or non-detrending is significantly different in the low-frequency part.

The Welch periodogram method based on the improved periodic graph method solves the above problem well, and it is also the most commonly used PSD method. The specific method steps are as follows:

(1) Segment the random sequence so that each piece of data has partial overlap.

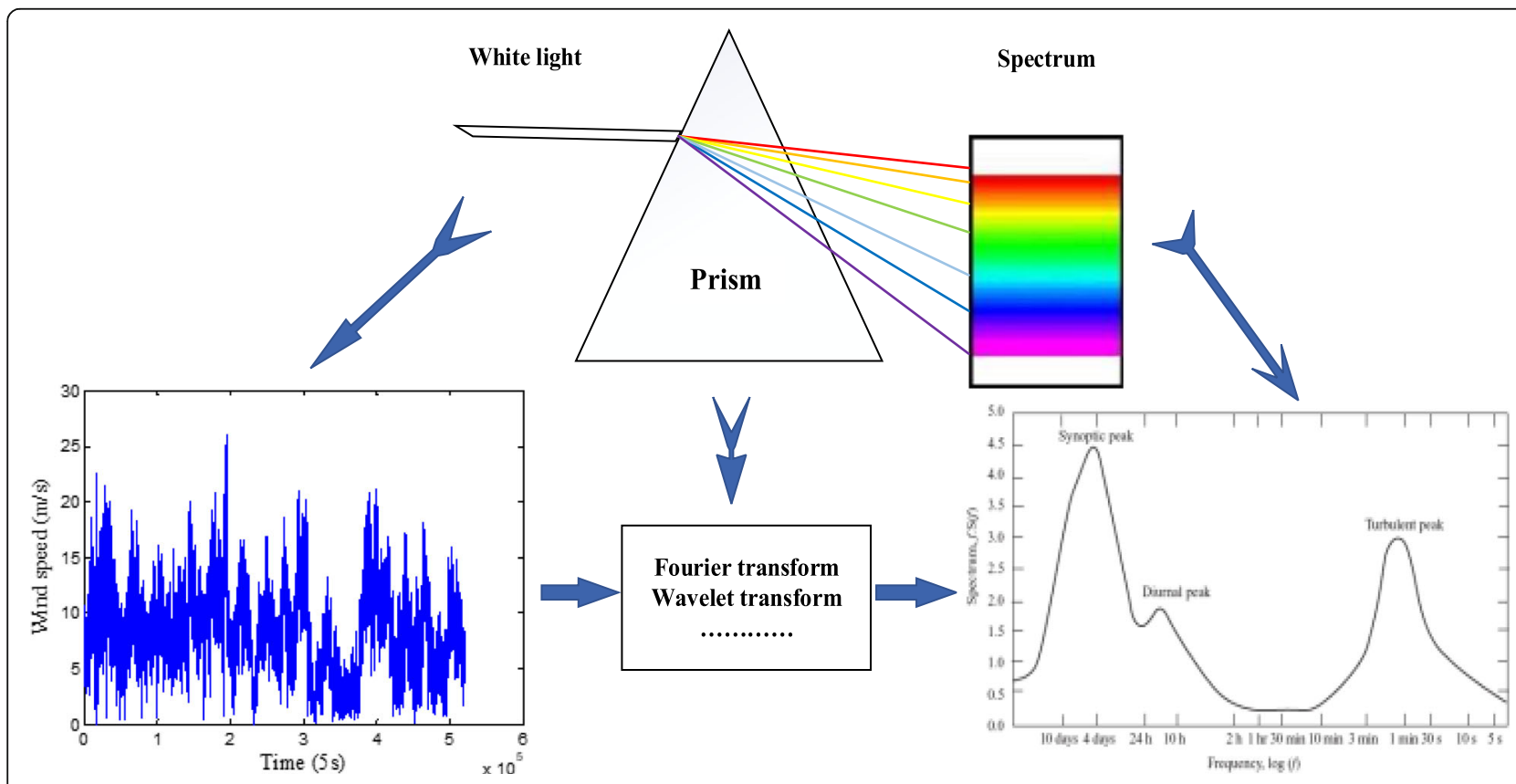

Fig. 1 Schematic of the spectral obtained using the characteristics analysis method 
Let $X(j), j=0, \ldots, N-1$ be a sample of a second-order random sequence. We take separate line segments at a starting point $L$ and length $L$, which may be overlapping. Let $X_{1}(j), j=0, \ldots, L-1$ be the first line segment; then, $X_{1}(j)=X(j), j=0, \ldots, L-1$; likewise, $X_{2}(j)=X(j+D), j=0, \ldots$, $L-1$. At last, $X_{K}(j)=X(j+(K-1) D), j=0, \ldots, L-1$. Suppose there are $K$ line segments: $X_{1}(j), \ldots, X_{K}(j)$, which cover the entire record, i.e., $(K-1) D+L=N$ as shown in Fig. 2.

(2) Smoothing each segment of data with a suitable window function and finally averaging the segments to obtain the desired power spectrum.

We calculate its modified periodogram for each length $L$. We select a data window $W(j), j=0, \ldots, L-1$ to form a sequence $X_{1}(j) W(j), \ldots, X_{K}(j) W(j)$. Furthermore, we take these sequences $A_{1}(n), \ldots, A_{K}(n)$ that are transformed by the finite Fourier.

Here,

$$
A_{k}(n)=\frac{1}{L} \sum_{j=0}^{L-1} X_{k}(j) W(j) e^{\frac{-2 k i n n}{L}}
$$

and $i^{2}=-1$. Finally, the correction period $K$ is obtained:

$$
I_{k}\left(f_{n}\right)=\frac{L}{U}\left|A_{k}(n)\right|^{2}, k=1,2, \ldots, K
$$

Here, $f_{n}=\frac{n}{L}, n=0, \ldots, \frac{L}{2}$, and $U=\frac{1}{L} \sum_{j=0}^{L-1} W^{2}(j)$.

The spectral estimate is the average of these periodic graphs, i.e.,

$$
\hat{P}\left(f_{n}\right)=\frac{1}{K} \sum_{k=1}^{K} I_{k}\left(f_{n}\right)
$$

Now, we can prove

$$
E\left\{\hat{P}\left(f_{n}\right)\right\}=\int_{-\frac{1}{2}}^{\frac{1}{2}} h(f) P\left(f-f_{n}\right) d f
$$

Here, $h(f)=\frac{1}{L U}\left|\sum_{j=0}^{L-1} W(j) e^{2 \pi i f f}\right|^{2}$ and $\int_{-\frac{1}{2}}^{\frac{1}{2}} h(f) d f=1$.

Thus, we obtain a spectral estimate $\hat{P}(f)$ whose area of the composite spectral window is uniform and the width is $1 / L$.

When using Fourier to analyze a random signal, the local features of the time domain signal cannot be characterized and the Gibbs effect is produced. At the same time, it does not work well for both the abrupt and nonstationary signals.

\subsection{Wavelet Mallat algorithm with compact support [36, 37]}

The wavelet transforms (WTs) can analyze the signal with a set of basic functions whose analysis width is constantly changing. This change adapts to the basic requirement that different resolutions are required in different frequency ranges for signal analysis. Moreover, wavelet analysis has the chrematistics of time domain and frequency domain localization, multiresolution, and adjustable time-frequency window, which is obviously an effective time-frequency multi-scale analysis tool for signal processing. Therefore, the envelope of the desired frequency range signal can be extracted by the wavelet envelope analysis method without the need of a filter. At the same time, the Mallat wavelet algorithm has more advantages than other wavelet algorithms.

Let $x(t) \in L^{2}(R), \psi(t) \in L^{2}(R)$, and $\psi(t)$ satisfy the admissible condition:

$$
C_{\psi}=\int_{-\infty}^{+\infty} \frac{|\hat{\psi}(\omega)|^{2}}{|\omega|} d \omega<+\infty
$$

Then, the continuous WT is defined as

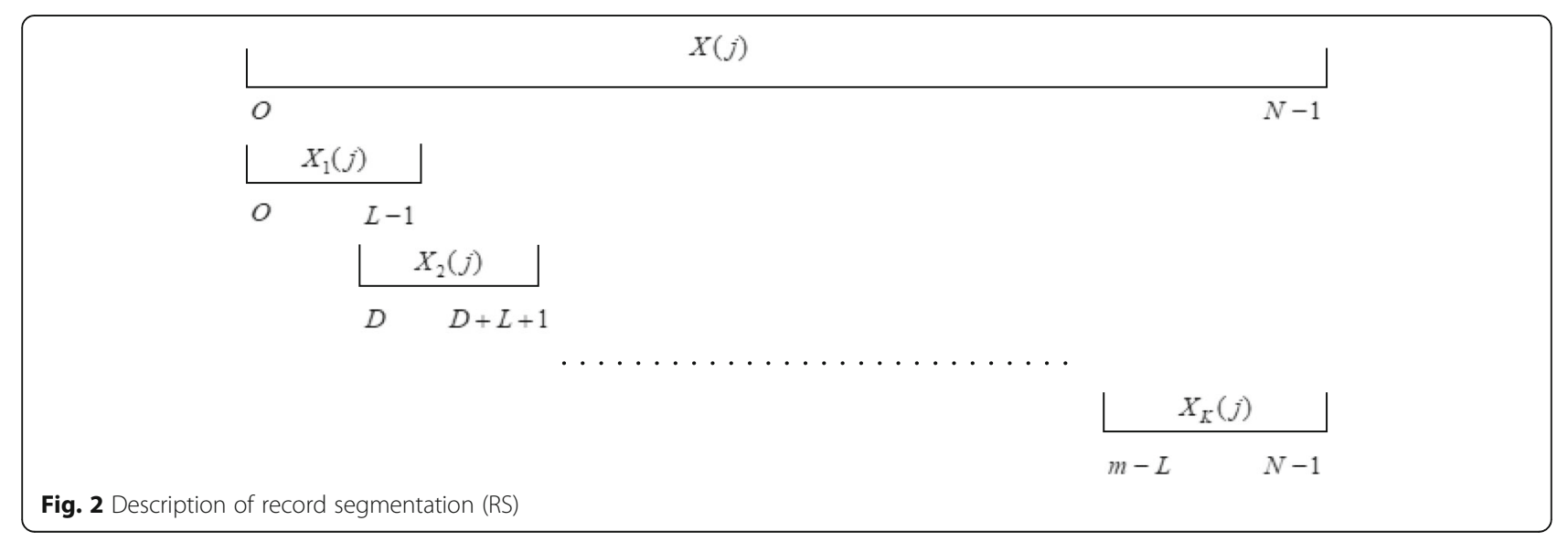




$$
\mathrm{WT}_{x}(a, b)=\int_{-\infty}^{+\infty} x(t) \bar{\psi}\left(\frac{t-b}{a}\right) d t, a \neq 0
$$

Or the inner product can be denoted as

$$
\mathrm{WT}_{x}(a, b)=\left\langle x, \psi_{a, b}\right\rangle
$$

where $a$ is the scale parameter, which controls the expansion and contraction of the wavelet function, and $b$ is the translation parameter, which controls the translation of the wavelet function. The scale $a$ corresponds to the frequency (inverse ratio) and the shift amount $b$ corresponds to the time. $\psi_{a, b}(t)$ meets $\psi_{a, b}(t)=\frac{1}{\sqrt{a}} \psi\left(\frac{t-b}{a}\right)$. In addition, $\psi_{a, b}(t)$ is finitely supported in the time domain. Then, the dot product of $\psi_{a, b}(t)$ and $s(t)$ is calculated. The WT is also finitely supported in time domain and achieves the time domain positioning function. $\hat{\psi}_{a, b}(\omega)$ has a band-pass characteristic, i.e., it is finitely supported around the center frequency in the frequency domain. Moreover, after calculating the dot product of $\hat{\psi}_{a, b}(\omega)$ and $S(\omega), S(\omega)$ will also reflect the local features at the center frequency of the window, thus achieving the desired frequency positioning function. Thus, we can obtain the instantaneous power spectrum characteristics of the signal.

When applying WT, we usually use the dispersion process. Simultaneously, in order to attain the resolution of time and frequency using the WT variable, it is necessary to change the values of $a$ and $b$. In practical applications, it is usually achieved by using binary dynamic sampling: $a_{0}=2, b_{0}=1$. Now, the discrete wavelet is

$$
\psi_{j, k}(t)=2^{-j / 2} \psi\left(2^{-j} t-k\right)
$$

This is the binary wavelet. However, the discrete wavelet still has a certain degree of redundancy. We require that the wavelet cluster $\left\{\psi_{j, k}\right\}$ can be an orthogonal basis, thus achieving non-redundant expansion and reconstruction. Frequency domain equal resolution is an inherent characteristic of short-time Fourier transform, and multi-resolution is an inherent characteristic of WT. However, the multi-scale analysis theory provides the most effective way to solve this problem. In simple terms, the multi-scale analysis theory is to represent the $x(t)$ function with a series of approximation function limits in space $L^{2}(R)$. A series of gradually refined approximation functions are obtained by the smooth approximation of function $x(t)$ under different scale conditions. Therefore, it is called multi-scale analysis.

Multi-scale analysis in space $L^{2}(R)$ can be understood by constructing a subspace sequence $\left\{V_{j}, j \in Z\right\}$ in space $L^{2}(R)$ that makes it have monotonicity $\left(V_{j} \in V_{j-1}, \forall j \in Z\right)$, approximation property $\left(\operatorname{close}\left\{\mathrm{U}_{j=-\infty}^{+\infty} V_{j}\right\}=L^{2}(R), \cap_{j=-\infty}^{+\infty}\right.$ $\left.V_{j}=\{0\}\right)$, elasticity $\left(\phi(t) \in V_{j} \Leftrightarrow \phi(2 t) \in V_{j+1}\right)$, translation invariance $\left(\phi(t) \in V_{j} \Leftrightarrow \phi\left(t-2^{j} k\right) \in V_{j} \quad k \in Z\right)$, and existence of Riesz bases $\left(\forall \phi(t) \in V_{0}\right.$, so that $\left\{\phi\left(t-2^{-j} k\right), k \in Z\right\}$ can constitute the Riesz base of $V_{0}$ ).

Theorem: If $V_{j}(j \in Z)$ represents an approximation of multiple scales in space $L^{2}(R)$, there must be a unique function $\phi(t) \in L^{2}(R)$ :

$$
\phi_{j, k}=2^{-j / 2} \phi\left(2^{-j} t-k\right), \quad k \in Z
$$

The function must represent a standard orthonormal basis in $V_{j} . \phi(t)$ is called a scaling function.

Simultaneously, define the wavelet subspace formed by the wavelet function $\psi\left(2^{-j} t\right)$ as

$$
W_{j}=\operatorname{close}\left\{\psi_{j, k}: k \in Z\right\}, j \in Z
$$

In the process of constructing an orthogonal wavelet basis, we should ensure that

$$
\begin{aligned}
& V_{j-1}=V_{j} \oplus W_{j}, \quad \forall j \in Z \\
& V_{j \perp} W_{j}
\end{aligned}
$$

It is true to all $j \in Z$. The $\oplus$ in the formula represents "orthogonal sum." Here, the subspaces $V_{j}$ and $W_{j}$ can be regarded as the complementary subspace of $V_{j-1}$. $W_{j}$ that represents the orthogonal complement of $V_{j}$ on $V_{j-1}$ is called wavelet space of scale $j$. It is clear that $V_{0}=$ $V_{1} \oplus W_{1}=V_{2} \oplus W_{2} \oplus W_{1}=\ldots=V_{N} \oplus W_{N} \oplus W_{N-1} \oplus$ $\cdots \oplus W_{2} \oplus W_{1}$. If $x_{j} \in V_{j}$ is an approximation of the function $x \in L^{2}(R)$ with a resolution of $2^{-j}$ and $d_{j} \in W_{j}$ is the approximation error, then the above equation can be expressed as

$$
\begin{aligned}
x_{0} & =x_{1}+d_{1}=x_{2}+d_{2}+d_{1}=\ldots \\
& =x_{N}+d_{N}+d_{N-1}+\ldots+d_{2}+d_{1}
\end{aligned}
$$

If $x \approx x_{0}$, the above formula can be written as

$$
x \approx x_{0}=x_{N}+\sum_{i=0}^{N} d_{i}
$$

This implies that any one of the signals $x \in L^{2}(R)$ can be completely reconstructed by the approximation of the signals and the approximation error of the signals at different scales.

The Mallat algorithm is based on the above ideas in multi-scale analysis. Assuming function $f(t) \in V_{j-1}$, the function can be expanded in space $V_{j-1}$ as

$$
f(t)=\sum_{k} c_{j-1, k} 2^{(-j+1) / 2} \phi\left(2^{-j+1} t-k\right)
$$

Decompose $f(t)$ and then project onto the $V_{j}$ and $W_{j}$ spaces. Thus, 


$$
\begin{aligned}
f(t)= & \sum_{k} c_{j, k} 2^{-j / 2} \phi\left(2^{-j} t-k\right) \\
& +\sum_{k} d_{j, k} 2^{-j / 2} \psi\left(2^{-j} t-k\right)
\end{aligned}
$$

$c_{j, k}$ represents the scale factor on the $j$ scale and $d_{j, k}$ represents the detail coefficient on the $j$ scale. In addition, the following conditions need to be satisfied

$$
\begin{aligned}
c_{j, k} & =\left\langle f(t), \phi_{j, k}(t)\right\rangle \\
& =\int_{R} f(t) 2^{-j / 2} \overline{\phi\left(2^{-j} t-k\right)} d t \\
d_{j, k} & =\left\langle f(t), \psi_{j, k}(t)\right\rangle \\
& =\int_{R} f(t) 2^{-j / 2} \overline{\psi\left(2^{-j} t-k\right)} d t
\end{aligned}
$$

After a series of derivations,

$$
\begin{aligned}
& c_{j, k}=\sum_{n} h(n-2 k) c_{j-1, n} \\
& d_{j, k}=\sum_{n} g(n-2 k) c_{j-1, n}
\end{aligned}
$$

This is the pyramid algorithm of the Mallat decomposition.

Similarly, the reconstruction algorithm of Mallat is

$$
c_{j-1, m}=\sum_{k} c_{j, k} h(n-2 k)+\sum_{k} d_{j, k} g(n-2 k)
$$

According to the above theory, the center frequency of the wind power signal can be obtained by using the compactly supported wavelet to extract the envelope.

And compared with the Fourier transform method, wavelet transforms the time and space-frequency localization analysis, which through the telescopic translation operations (functions) of signal gradually multiscale refinement. Ultimately, it achieves high-frequency time segment and the low frequency in the frequency segment, which can automatically adapt to the requirement of time-frequency signal analysis. And it focuses on the arbitrary signal details to solve the difficult problem of Fourier transform. Therefore, wavelet transform becomes an important breakthrough in scientific methods following Fourier transform.

\section{Discussion and results}

\subsection{Instantaneous spectral characteristic extraction of wind power}

We use the 1-year measured data of a wind farm in Inner Mongolia, China. The wind farm has 100 wind turbines of Vestas V80-2000 with a rated power of 2000 $\mathrm{kW}$, and the sampling period is $5 \mathrm{~s}$.
First, based on the output power signal of the single wind turbines, we performed conventional power spectral density estimation and extracted the power spectrum with instantaneous characteristics of the signal. As shown in Fig. 3, the result we achieved is similar to the result we obtained using the Welch periodogram algorithm (based on the Fourier transform algorithm). Moreover, the result is in agreement with the classic Kolmogorov "-5/3 law" [28]. Therefore, it shows the rationality of extracting the instantaneous spectral characteristics of wind power signals based on the compactly supported wavelet Mallat algorithm. We analyzed the data of several other wind turbines in this wind farm and obtained consistent results.

On this basis, we obtained the law for the change in center frequency and average power, as shown in Fig. 4. It can be seen that the center frequency tends to be constant as the power of the wind turbine increases. Furthermore, power spectral density estimation is performed on the measured wind power of all wind turbines of the wind farm and the obtained results are consistent. It shows that this characteristic is also adapted to the entire farm power signal.

\subsection{Time and frequency separation characteristics of wind power}

Similarly, we explore the joint characteristics of wind power in the time and frequency domains because few people associate the time domain and frequency domain of wind power. We used the multi-resolution wavelet Mallat algorithm to analyze the measured wind power data in the time domain. We could obtain the dependents between the uncertainty part and average power in the wind power signal, which can be used to describe the quantitative characterization model $[14,15]$ :

$$
I_{w}=\frac{\sigma_{w}}{\bar{W}}
$$

Here, $\sigma_{w}$ represents the standard deviation of wind power and $\bar{W}$ represents the average wind power. $I_{w}$ represents the fluctuation range of real-time wind power fluctuations near the average of the wind power output.

As showed in Fig. 5, we will achieve a single-machine (full-farm turbines) three-parameter power law universal model by the result based on power generation signals of multiple turbines. Moreover, as the average power of single (full-field) wind turbine increases, the wind power fluctuation intensity tends to substantially be unchanged. And The detailed data processing and calculation fitting process can refer to $[14,15]$.

It can be seen from Figs. 3 and 4 that the wind power has a time-frequency separation characteristic, which can be described by the following formula: 


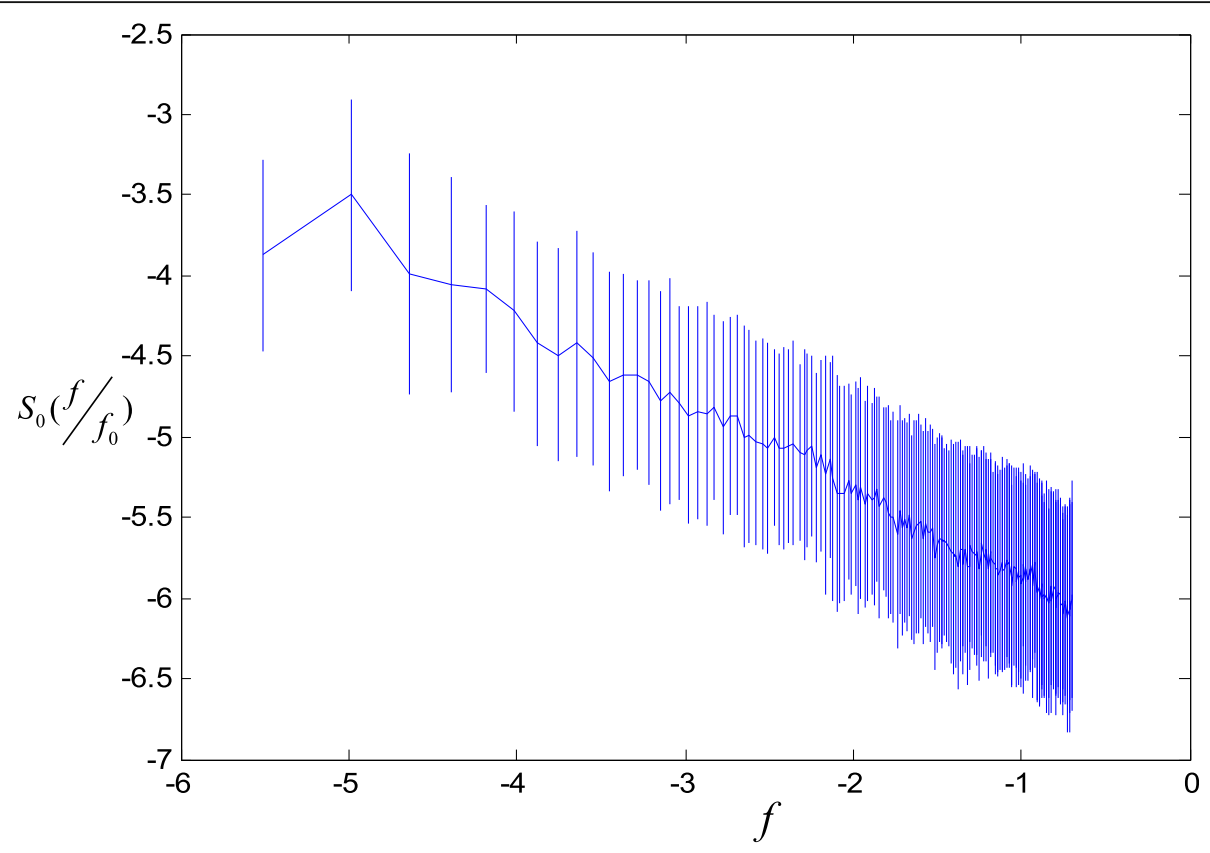

Fig. 3 Energy spectrum of wind power

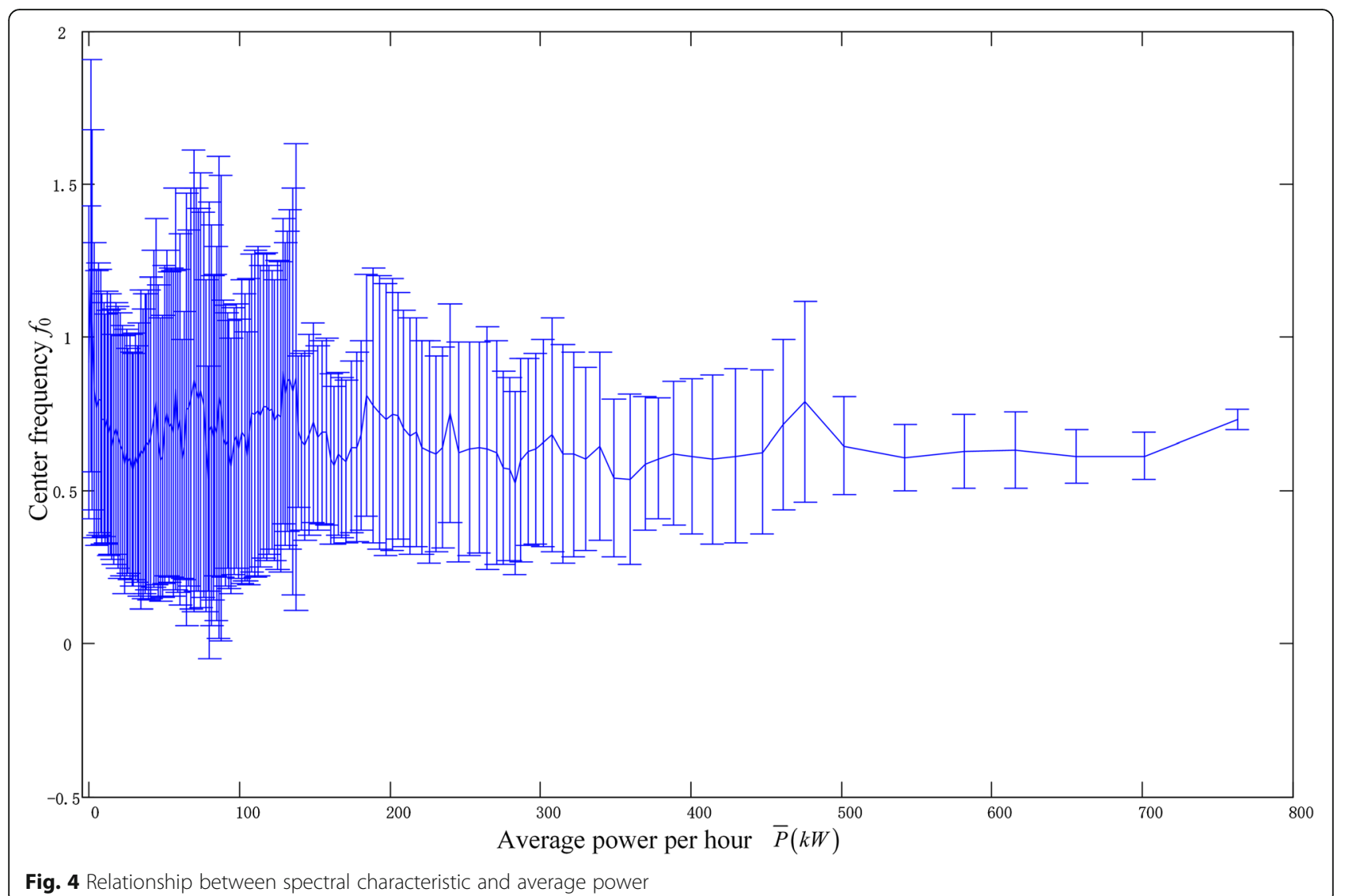

Fig. 4 Relationship between spectral characteristic and average power 


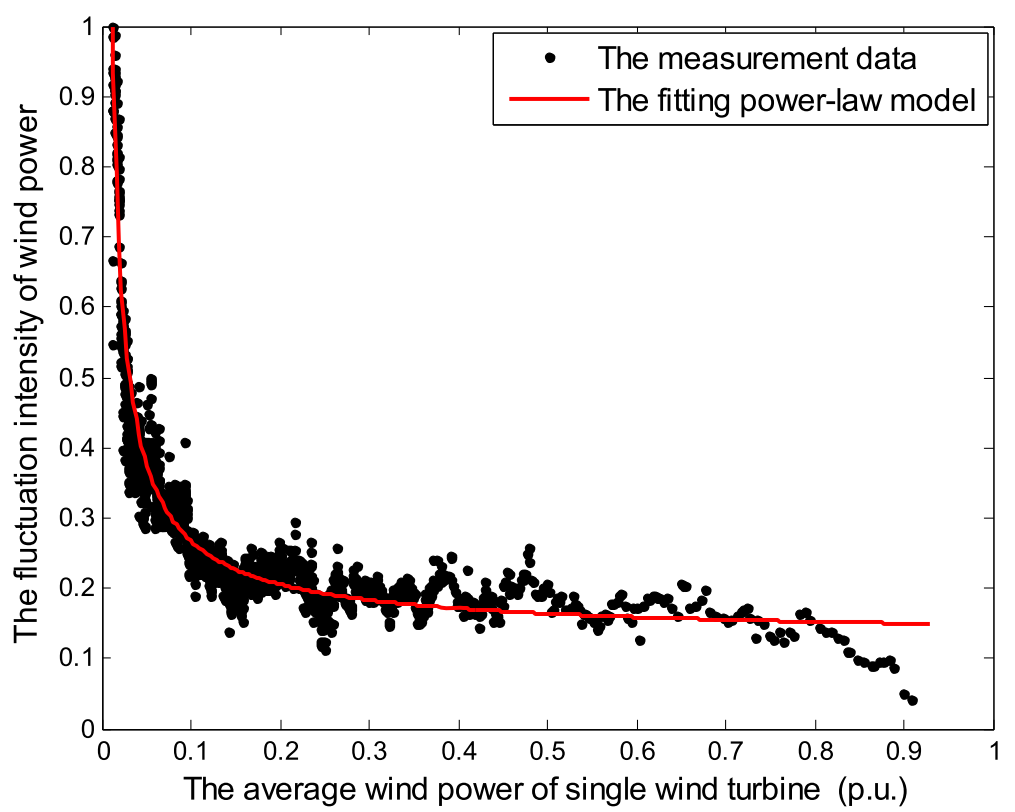

Fig. 5 Relationship between wind power uncertainty and the average value

$$
S(f, t)=\sigma^{2}[\bar{P}(t)] S_{0}\left(f / f_{0}\right)
$$

$f$ is the real-time frequency of wind speed, $t$ is the time, and $f_{0}$ is the center frequency.

The wind power spectrum characteristics have many practical applications, one of which is shown in [15]. However, in [15], the conventional statistical power spectrum is used. In addition, the application of instantaneous power spectrum can be studied.

\subsection{Energy conversion mechanism of wind turbines}

As shown in Fig. 6, the wind turbine power signal is an energy conversion made by the wind speed driven by complex turbulence. Therefore, we must first understand the spectral characteristics of wind speed in order to understand its physical mechanism.

According to the above method, we analyzed the four quarters of the wind speed data of this wind farm and obtained the dependency relationship between the hourly average wind speed and the wind speed of the turbulence. Furthermore, we analyzed the instantaneous

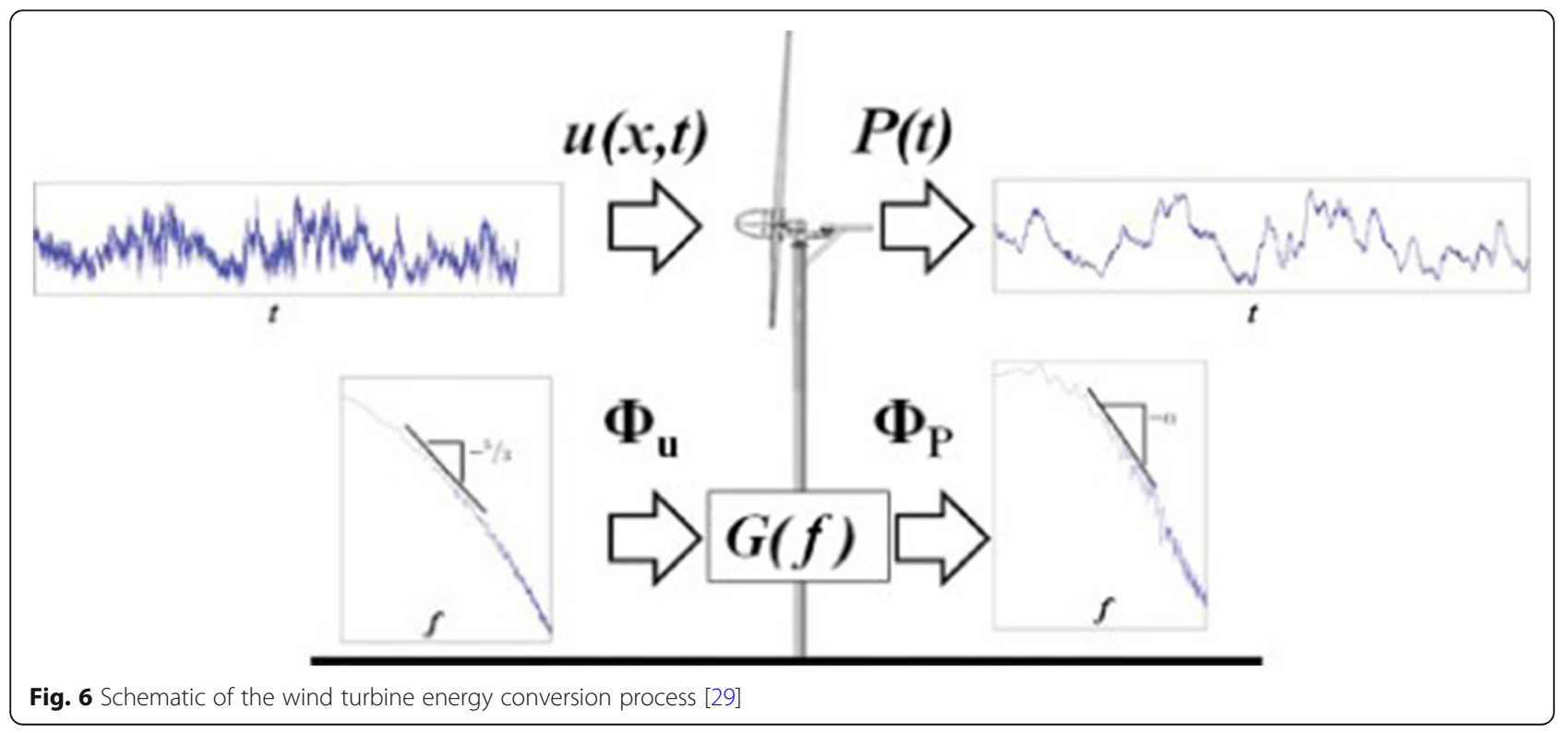



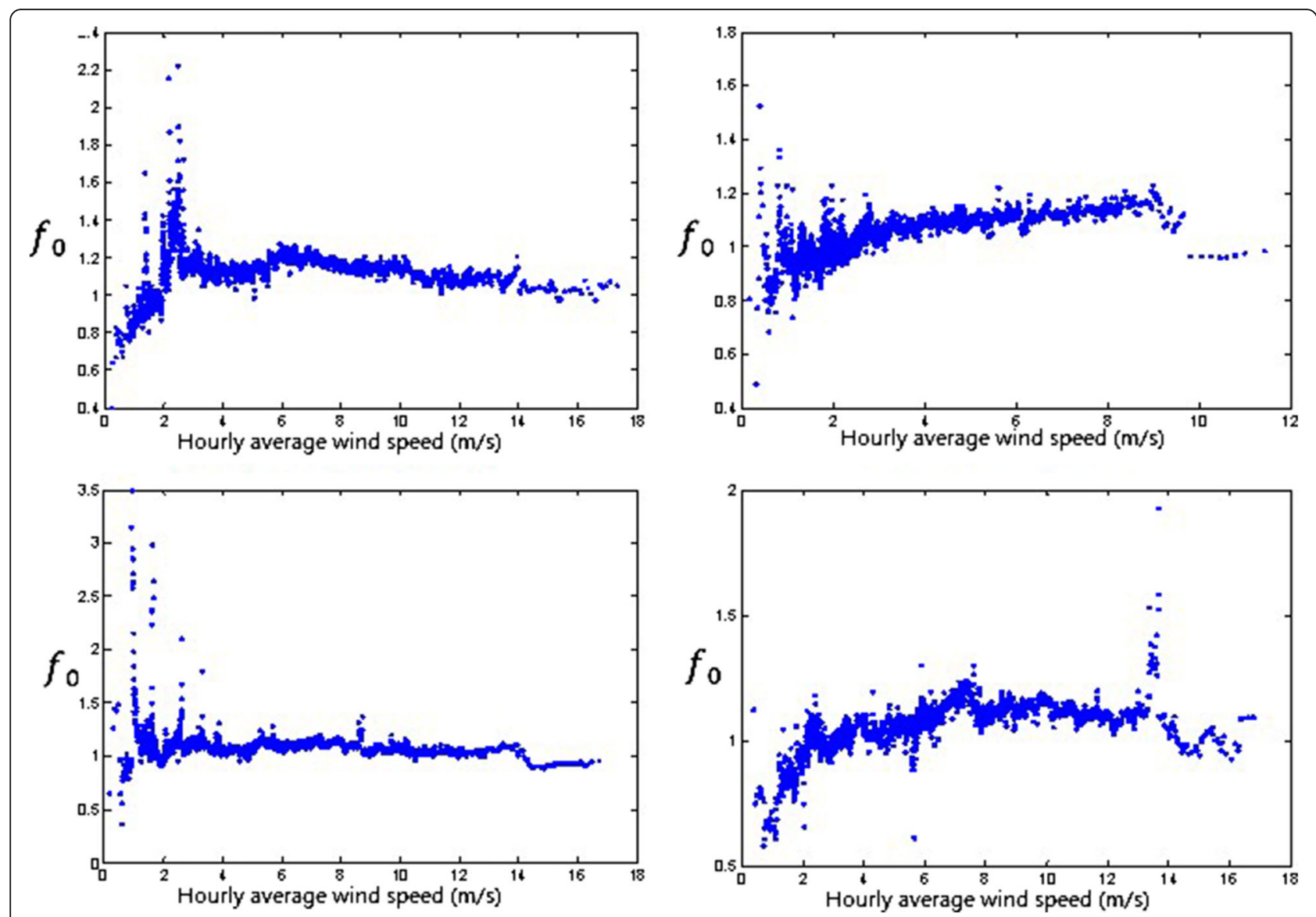

Fig. 7 Relationship between spectral characteristic and average wind speed

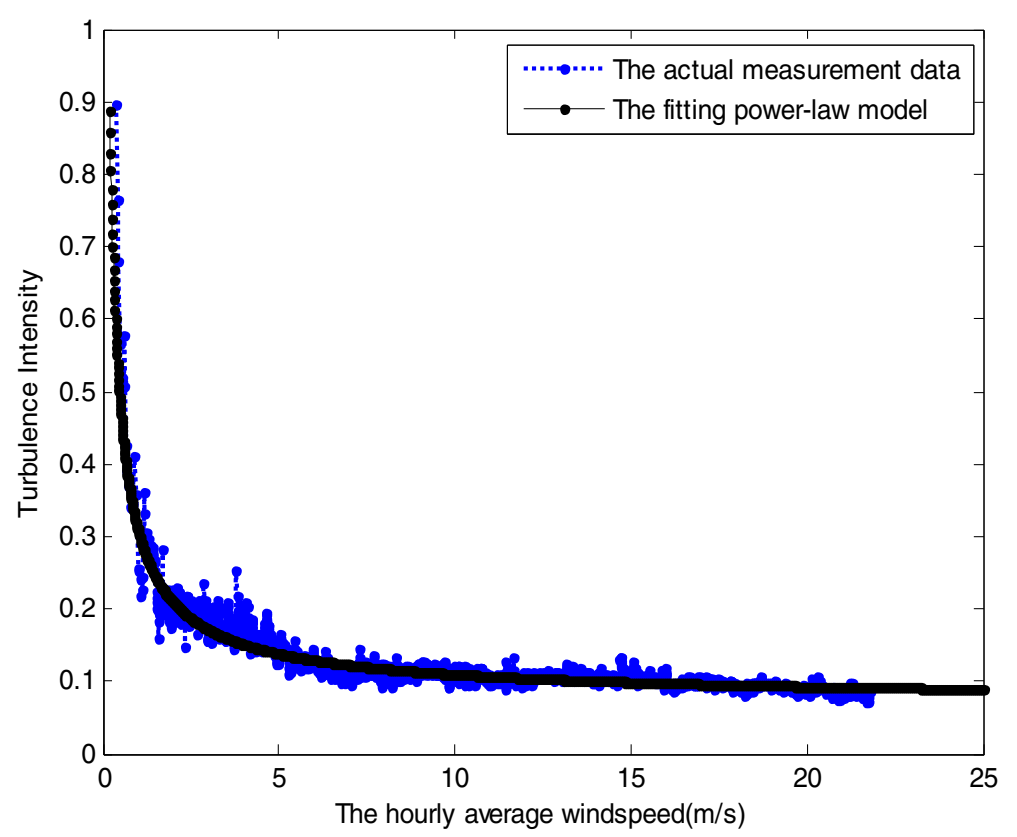

Fig. 8 Relationship between wind speed's average value and its corresponding fluctuation part 


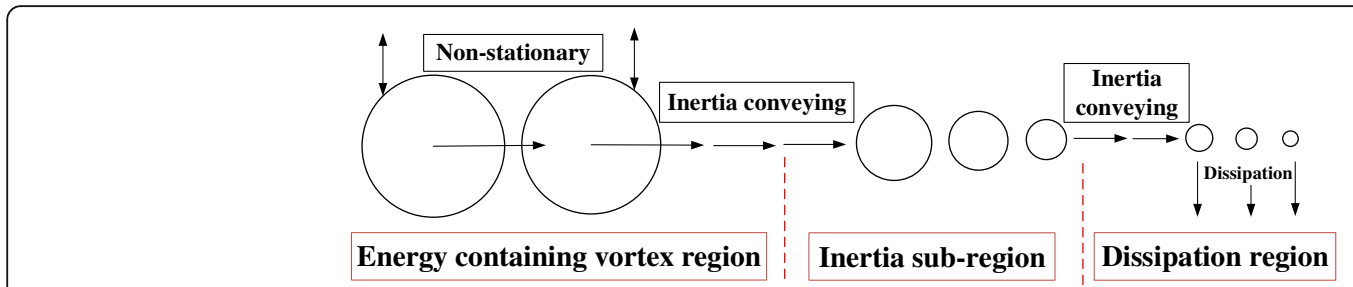

Fig. 9 Cascade transport mechanism of turbulence flow energy $[39,40]$

spectrum characteristics of the wind speed and obtained the relationship between the center frequency and the hourly average wind speed, as shown in Fig. 7. Within a certain range, the center frequency of the wind speed increases as the wind speed increases. However, the center frequency $f_{0}$ remains unchanged when the wind speed is sufficiently large. That is to say, $f_{0}$ also has the modulation effect, which is defined as frequency modulation (FM) effect.

In fact, based on the research of Leithead, W.E, Welfonder used the wind speed data of two wind fields for one month each to further improve the spectrum characteristic model and noticed the influence of the average wind speed on frequency, i.e., as the wind speed increases, the frequency also increases [38].

$$
G_{F}(i \omega)=\frac{V_{F}}{\left(1+i \omega \widehat{T}_{F}\right)^{5 / 6}}
$$

Here, $V_{F} \approx \sqrt{\frac{2 \pi}{B\left(\frac{1}{2} \cdot \frac{1}{3}\right)} \frac{\widehat{T}_{F}}{T}}, \widehat{T}_{F}=\frac{L}{\bar{v}_{\omega}}$, and $\bar{v}_{\omega}$ represent the average wind speed, $L$ represents the scale of turbulent length, $\mathrm{B}\left(\frac{1}{2}, \frac{1}{3}\right)$ is the specified beta function, $T$ is the finite sampling time, and $f_{0}=1 / \widehat{T}_{F}$ is the center frequency.

Therefore, the wind speed has local characteristics, and the spectral characteristics have special characteristics because of the influence of the complex underlying surface. This is closely related to the energy transport mechanism of atmospheric turbulence. As the wind turbine has cutin and cutoff wind speeds, the center frequency and average of the wind speed change characteristic, as shown in Fig. 7. And the actual complex conditions changed the turbulence structure. As a result, the wind turbine can output the power signal characteristics, as shown in Fig. 4, by conversion of wind turbine energy, as shown in Fig. 6.

However, the relationship between wind speed's average value and its corresponding fluctuation part in Fig. 8

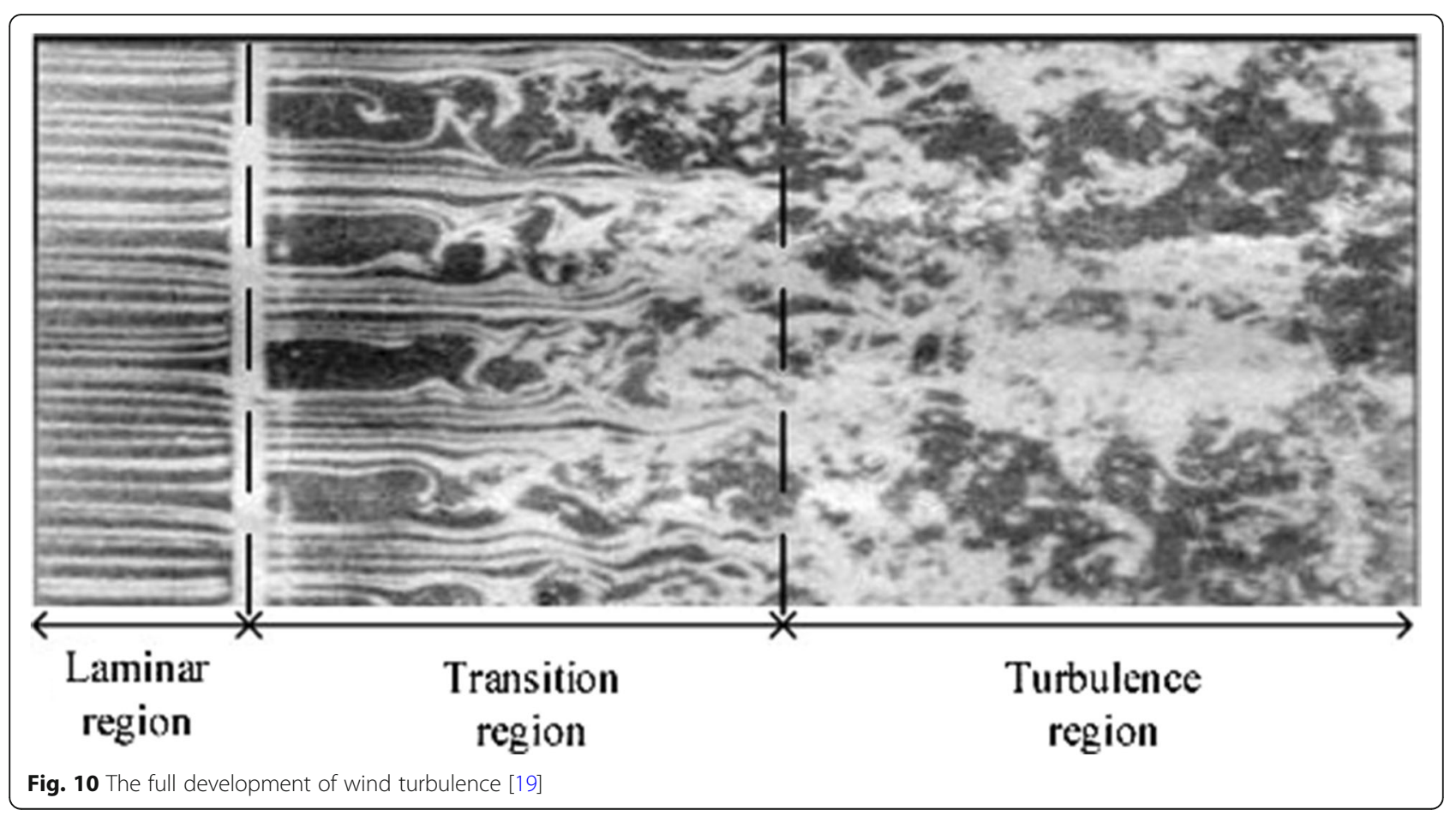


does not change by conversion of wind turbine energy shown in Fig. 6. And there is an amplitude modulation (AM) relationship between wind power's average value and its corresponding uncertainty in Fig. 5. So, the separation characteristics between time domain and frequency domain of wind power signal corresponds to the amplitude and frequency modulation characteristics of wind speed in wind power farm.

In addition, the whole wind farm data calculating results show that wind farm power output also has similar AM character. In the future, more cases will be further studied from the perspective of full farm power.

\subsection{Atmospheric turbulent energy transport mechanism}

In general, the spectrum of the turbulent motion of the atmospheric boundary layer includes the energetic region of large-scale turbulence, the inertial sub-region of small-scale turbulence, and the dissipative region. The uncertainty of wind speed fluctuations is caused by turbulence. Turbulence consists of eddies with large differences and various scales. The energy of the largest scale turbulence eddy region comes directly from the Reynolds stress work in the mean flow field and the buoyancy work in the atmospheric boundary layer. The energy obtained from outside by the large-scale turbulence eddy is transferred to the secondary turbulence eddy in stages and finally dissipated on the smallest scale turbulence eddy. In the process of cascade transmission, the smallscale vortices reach a statistical equilibrium state and no longer depend on the external conditions that generate turbulence, and thus form a so-called local uniform isotropic turbulence. Figures 9 and 10 show the cascade transport mechanism of turbulence energy flow, and the downward arrow indicates the dissipation of the turbulent energy.

Therefore, the time-frequency separation characteristics of the wind power signal are derived from the transmission and conservation of turbulence energy.

\section{Conclusions}

The power spectrum characteristics of the active power of wind power are an important manifestation of the wind power fluctuation characteristics. In addition, the instantaneous power spectrum characteristics are important for real-time scheduling and optimal control of new energy power systems. We studied the relationship between the time domain and frequency domain of wind power by calculating and analyzing the measured data of the wind farm. The following conclusions were observed:

(1) The Mallat algorithm based on wavelets with compact support realized the instantaneous spectral characteristics of wind power big data. Moreover, the obtained power spectrum is in agreement with Kolmogorov's " - 5/3 law."

(2) We observed variations in the center frequency as the wind power changes; the center frequency tends to be constant as the wind turbine power increases. Combining the dependence of the wind power mean and the variance of the opposite, we obtained the time-frequency separation characteristics of wind power and gave an explanatory expression. And the wind power's separation characteristics between time domain and frequency domain correspond to wind speed's amplitude and frequency modulation characteristics.

(3) Combined with the dual mechanisms of wind turbine energy conversion and atmospheric turbulence operation, we obtained the physical connotation of wind power time-frequency separation. As the atmospheric turbulence is increasing within a certain wind speed range, the center frequency increases with the increase of wind speed. However, when the wind speed is sufficiently large, i.e., when the atmospheric turbulence increases to a certain extent, the center frequency remains basically unchanged.

In the future, we can study the spectral characteristics of wind farms in different regions with varying latitudes and longitudes because the energy transfer mechanism of atmospheric turbulent is significantly affected by the latitude and longitude, underlying surface, and topography. Thus, it lays a foundation for the safe and efficient grid-connected operation of large-scale wind power.

\section{Abbreviations}

AM: Amplitude modulation; AR: Auto regressive; FFT: Fast Fourier transform; FM: Frequency modulation; PM: Phase modulation; PSD: Power spectrum density; RS: Record segmentation; WT: Wavelet transform

\section{Acknowledgements \\ We thank the editor and anonymous reviewers for their helpful comments and valuable suggestions. I would like to acknowledge all our team} members. They contributed equally to this work.

\section{Authors' contributions}

JW formulated the idea. All authors take part in the discussion of the work described in this paper. KY and PE contributed equally to this work and should be considered co-correspondent authors. All authors read and approved the final manuscript.

\section{Funding}

This work was financially supported through grants from the Key R\&D Project of China under grant number 2016YFB0901900, the State Key Laboratory of Alternate Electrical Power System with Renewable Energy Sources (grant no. LAPS19012), CERNET Innovation Project (no. NGIICS20190801), and the Natural Scientific Research Innovation Foundation in Harbin Institute of Technology of no. HIT.NSRIF.2020044.

Availability of data and materials

The datasets used and/or analyzed during the current study are available from the corresponding author on reasonable request. 


\section{Competing interests}

The authors declare that they have no competing interests.

\section{Author details}

${ }^{1}$ Fundamental Space Science Research Center, Harbin Institute of Technology, Harbin 150001, Heilong, China. ${ }^{2}$ Postdoctoral Research Station of Electrical Engineering, Harbin Institute of Technology, Harbin 150001, Heilong, China. ${ }^{3}$ Shenzhen Research Institute, The University of Hong Kong, Shenzhen 518057, Guangdong, China. ${ }^{4}$ School of Mechanical Engineering and Automation, Harbin Institute of Technology, Shenzhen 518055, China. ${ }^{5}$ The State Key Laboratory of Alternate Electrical Power System with Renewable Energy Sources (North China Electric Power University), Beijing 102206, China. ${ }^{6}$ School of Electrical Engineering \& Automation, Harbin Institute of Technology, Harbin 150001, Heilong, China.

Received: 12 July 2019 Accepted: 4 October 2019

Published online: 03 January 2020

\section{References}

1. M. Kenisarin, V.M. Karslı, M. Çağlar, Wind power engineering in the world and perspectives of its development in Turkey. Renew. Sust. Energ. Rev. 10(4), 341-369 (2006)

2. A.A. Juárez, A.M. Araújo, J.S. Rohatgi, Development of the wind power in Brazil: political, social and technical issues. Renew. Sust. Energ. Rev. 39(6), 828-834 (2014)

3. Z He, S Xu, W Shen, et al. Overview of the development of the Chinese Jiangsu coastal wind-power industry cluster. Renewable and Sustainable Energy Reviews, 57: 59-71(2016),

4. G. Callegari, P. Capurso, F. Lanzi, et al., Wind power generation impact on the frequency regulation: study on a national scale power system. Wind Energy, 1-6 (2010)

5. A S Brouwer, M Van Den Broek, A Seebregts, et al. Impacts of large-scale Intermittent Renewable Energy Sources on electricity systems, and how these can be modeled. Renewable and Sustainable Energy Reviews, 33 : 443-466(2014)

6. G. Ren, J. Liu, J. Wan, et al., Overview of wind power intermittency: impacts, measurements, and mitigation solutions. Appl. Energy 204, 47-65 (2017)

7. W. Wang, S. Jing, Y. Sun, et al., Combined heat and power control considering thermal inertia of district heating network for flexible electric power regulation. Energy 169, 988-999 (2019)

8. X. Li, Y. Zou, J. Wan, et al., Study on failure characteristics and solutions for large turbine load oscillation. 2016 IEEE Advanced Information Management, Communicates, Electronic and Automation Control Conference (IMCEC) (IEEE, 2016), pp. 1321-1325

9. L. Lu, H. Yang, J. Burnett, Investigation on wind power potential on Hong Kong islands - an analysis of wind power and wind turbine characteristics. Renewable Energy 27(1), 1-12 (2014)

10. J.A. Carta, S. Velázquez, A new probabilistic method to estimate the longterm wind speed characteristics at a potential wind energy conversion site. Energy 36(5), 2671-2685 (2011)

11. S.P. Rensen, A.D. Hansen, P.A.C. Rosas, Wind models for simulation of power fluctuations from wind farms. J. Wind Eng. Ind. Aerodyn. 90(12-15), 13811402 (2002)

12. P. Sorensen, N.A. Cutululis, A. Viguerasrodriguez, et al., Power fluctuations from large wind farms. IEEE Trans. Power Syst. 22(3), 958-965 (2007)

13. P. Sørensen, N.A. Cutululis, A. Vigueras-Rodríguez, et al., Modelling of power fluctuations from large offshore wind farms. Wind Energy 11(1), 29-43 (2008)

14. G. Ren, J. Liu, J. Wan, et al., The analysis of turbulence intensity based on wind speed data in onshore wind farms. Renewable Energy 123, 756-766 (2018)

15. Y. Guo, Q. Wang, D. Zhang, et al., A stochastic-process-based method for assessing frequency regulation ability of power systems with wind power fluctuations. J. Environ. Inf. (2018)

16. J. Liu, G. Ren, J. Wan, et al., Variogram time-series analysis of wind speed. Renewable Energy 99, 483-491 (2016)

17. X. Yi, X. Zha, Q. Liang, et al., Research on wind power ramp events prediction based on strongly convective weather classification. let Renew. Power Generation 11(8), 1278-1285 (2017)

18. C. Gallego, A. Costa, Á. Cuerva, et al., A wavelet-based approach for large wind power ramp characterisation. Wind Energy 16(2), 257-278 (2013)
19. G. Ren, J. Liu, J. Wan, et al., Measurement and statistical analysis of wind speed intermittency. Energy 118, 632-643 (2017)

20. G. Ren, J. Wan, J. Liu, et al., Analysis of wind power intermittency based on historical wind power data. Energy 150, 482-492 (2018)

21. Q. Hu, S. Zhang, M. Yu, et al., Short-term wind speed or power forecasting with heteroscedastic support vector regression. IEEE Transactions on Sustainable Energy 7(1), 241-249 (2017)

22. R Girard, D Allard. Spatio-temporal propagation of wind power prediction errors. Wind Energy, 16(7): 999-1012(2013).

23. Y. Jie, Y. Liu, H. Shuang, et al., Reviews on uncertainty analysis of wind power forecasting. Renew. Sust. Energ. Rev. 52, 1322-1330 (2015)

24. M.G.D. Giorgi, A. Ficarella, M. Tarantino, Error analysis of short term wind power prediction models. Appl. Energy 88(4), 1298-1311 (2011)

25. A.N. Kolmogorov, Dissipation of Energy in Locally Isotropic Turbulence. Akademiia Nauk Sssr Doklady 32(1890), 15-17 (1941)

26. D. Ruelle, F. Takens, On the nature of turbulence. Commun. Math. Phys. 20(3), 167-192 (1971)

27. J.C. Kaimal, L. Kristensen, Time series tapering for short data samples. Boundary Layer Meteorol. 57(1-2), 187-194 (1991)

28. J. Apt, The spectrum of power from wind turbines. J. Power Sources 169(2), 369-374 (2007)

29. N. Tobin, H. Zhu, L.P. Chamorro, Spectral behaviour of the turbulence-driven power fluctuations of wind turbines. J. Turbul. 16(9), 832-846 (2015)

30. J. Bossuyt, C. Meneveau, J. Meyers, Wind farm power fluctuations and spatia sampling of turbulent boundary layers. J. Fluid Mech. 823, 329-344 (2017)

31. S. Frandsen, M.L. Thøgersen, Integrated fatigue loading for wind turbines in wind farms by combining ambient turbulence and wakes. Wind Eng., 327339 (1999)

32. K.M. Talluru, R. Baidya, N. Hutchins, I. Marusic, Amplitude modulation of all three velocity components in turbulent boundary layers. J. Fluid Mech. 746 (2014)

33. S.T. Frandsen, Generated structural loading in wind turbine clusters (2007)

34. D. Mencarelli, A. Di Donato, T. Rozzi, Analytical study of the optical spectrum shift in a modulating channel. J. Lightwave Technol. 24(2), 1035 (2006)

35. P. Welch, The use of fast Fourier transform for the estimation of power spectra: a method based on time averaging over short, modified periodograms. IEEE Trans. Audio Electroacoust. 15(2), 70-73 (1967)

36. R.S. Pathak, The Wavelet Transform (Atlantis Press, 2009)

37. I. Daubechies, The wavelet transform, time-frequency localization and signal analysis. J Rene. Sust. Energy. 36(5), 961-1005 (2015)

38. E. Welfonder, R. Neifer, M. Spanner, Development and experimental identification of dynamic models for wind turbines. Control. Eng. Pract. 5(1), 63-73 (1997)

39. S.B. Pope, Turbulent flows (Cambridge university press, 2000)

40. J C Kaimal, J J Finnigan. Atmospheric boundary layer flows: their structure and measurement. Oxford university press, 1994

\section{Publisher's Note}

Springer Nature remains neutral with regard to jurisdictional claims in published maps and institutional affiliations.

\section{Submit your manuscript to a SpringerOpen ${ }^{\circ}$ journal and benefit from:}

- Convenient online submission

- Rigorous peer review

- Open access: articles freely available online

High visibility within the field

- Retaining the copyright to your article

Submit your next manuscript at $\boldsymbol{\sim}$ springeropen.com 